${ }^{1}$ Department of Botany and Ecology, University of Technology and Life Sciences in Bydgoszcz, Prof. S. Kaliskiego 7, 85-796 Bydgoszcz, Poland; ${ }^{2}$ Department of Grasses, Legumes and Energy Plants, Plant Breeding and Acclimatization Institute - National Research Institute, Radzików, $05-870$ Błonie, Poland

\title{
ESTIMATION OF ROOT AND SHOOT GROWTH OF SELECTED GRASS SPECIES ON THE BASIS OF SEEDLING CHARACTERIZATION
}

\begin{abstract}
Our study was designed to describe possibilities to estimate above- and below ground biomass of selected grass species by means of seedling development. Festuca arundinacea Schreb., Festuca rubra L., Lolium perenne L., Poa pratensis L., Koeleria macrantha (Ledeb.) Schultes, Deschampsia caespitosa (L.) P.B. were used in our experiment. On the basis of observations and measurements, differences between species, cultivars and ecotypes were described. Significant and positive correlation was found between root:shoot ratio at seedling phase and at mature plants.
\end{abstract}

Key words: turf grass, root system, aboveground biomass

\section{INTRODUCTION}

Effective use of turf grass is strictly connected with good species and variety selection made on the basis of morphological and biological features as well as site requirements (Prończuk 1994). Good lawn is usually a mixture of different species and varieties therefore selection of mixture components is very important for lawn quality. And it is not easy not only due to different growth and development patterns of grass species and cultivars, but also different reaction to climatic and soil conditions (Rutkowska and Hempel 1986, Grabowski et al. 1999a). Although intensive turf re-

Communicated by Andrzej Aniot 
search performed in USA, Europe etc. (some literature cited in Waddington et al. 1992, Sherman 2006) recently published articles focused on this topic are not numerous in Poland. Articles published so far were mostly connected with numerous traits observed on well developed turf surfaces. Traits as visual merit, uniformity, shoots density, turf color, leaf fineness were observed and described for range of species and varieties (Waddington et al. 1992, Prończuk 1993, Prończuk et al. 1997, Grabowski et al. 1999, Żurek et al. 2001, Jankowski et al. 2003, Prończuk and Żurek 2008). Some effort has been paid also on grass growth dynamics at initial stage of plant development (Harkot 1998, Harkot 2006) or reaction to different site conditions as: drought (Żurek 2004, 2006), shade (Prończuk et al. 2003), frequency of mowing (Stuczyńska and Jakubowski 1980), fertilization and organic matter (Rutkowska and Pawluśkiewicz 1996, Dziamski et al. 2007, Tołwińska 1975). Research on grass root system, which is very important in plant life, with its critical role in survival of stress conditions, is also rather limited (Tołwińska 1975, Harkot and Czarnecki 1998, Harkot 2006, Prończuk and Żurek 2008, Dziamski i wsp. 2008). Differences among plant species in root system growth are assumed to influence their ability to acquire resources (water, nutrients etc.) and, consequently, their competitive ability (Gross et al. 1992). Breeders, scientists and turf grass 'end-users' often search for good criterion for prediction of mature plant growth and development on the basis of seedling observation and selection. As seedling development undergoes successive stages (i.e. unimbibed seed, radicle and coleoptile emergence, seminal roots growth and elongation etc.) many of mentioned traits are highly heritable (Gibson, 2009). It is also possible that some of mentioned traits may be reflected during next stages of plant growth.

The aim of our study was to find relations between seedlings and mature plants of six turf grass species on the basis of below- and aboveground biomass yields.

\section{MATERIALS AND METHODS}

Following entries of six turf grass species were selected for our study: Festuca arundinacea Schreb. - varietes: Rahela, WOM -100 and Asterix, Festuca rubra L. - ecotypes: POLLBS-263, POLLBS-578 and POLLBS-393, Lolium perenne L. - varietes: Lisuna, Numan and Gator, Poa pratensis L.- varietes: Chałupy, Srzeszowicka and ecotype POLLBS-587, Deschampsia caespitosa (L.) P.B. - variety Brok and ecotype POLLBS-236, Koeleria macrantha (Ledeb.) Schultes - ecotype UKR 119. Entries were selected to cover wide range of grass species suitable for turf. Despite of varieties and breeding strains of well known high turf quality, ecotypes used were of previously confirmed turf potential (Schmidt, personal communication). Deschampsia caespitosa and Koeleria macrantha are currently very promising for turf purposes, but there is lack of available seed material, therefore in case of this species only few, currently accessible accessions were used. 
Experiment was divided into two phases: seedling phase (emergence of $6-10$ leaves) and mature plants phase (up to 2.5 years old or 945 days since seed sowing).

Seed was sown in March of 2006 in soil mixture of 1 part of compost soil, 1 part of peat and 1 part of sand. Soon after seed emergence, seedlings were transferred to small pots of volume $0.2 \mathrm{dm}^{3}$. Fifty pots per entry were further placed in unheated glass house (mean air temperature $15^{\circ} \mathrm{C}$, water added as necessary) until they reached phase of 6-10 leaves. Depending of species it took 35 to 40 days from seed sowing. From 15 seedlings per entry ( 5 plants per 3 replications) above-ground biomass (further referred as to shoots), was cut off, dried and weight. Below-ground biomass (further referred as to roots) from pots were washed in water and also dried and weight.

In mid July of 2006, eighteen plants from each entry were randomly selected and transferred to metal pots $\left(5000 \mathrm{~cm}^{3}, 20 \mathrm{~cm}\right.$ in diameter, with drainage hole at the bottom, filled with $6.0 \mathrm{~kg}$ of soil mixture, same as for seed emergence, one plant per one pot). For the rest of test duration plants were grown outside, frequently watered as necessary and hand mowed, with clippings collected, dried and weight. Pots were mown three times in 2007 and four times in 2008. Each year in April and July pots were fertilized ( $30.9 \mathrm{~kg} \mathrm{~N}, 16.7 \mathrm{~kg} \mathrm{P}, 49.6 \mathrm{~kg} \mathrm{~K}$ and $5.3 \mathrm{~kg} \mathrm{Mg}$ ). Each year at the beginning (March), mid (July) and end (October) of growing season three pots per entry were randomly selected. Plants were cut off and roots were washed in water, dried and weight. For each from above mentioned terms, shoot dry weight were calculated as sum of all previous cuts and the current one.

Results were further analyzed with SAS ${ }^{\circledR}$ statistical package. Fisher test (HSD) was used to calculate LSD values.

Weather conditions (monthly mean air temperature and total rainfall) were analyzed according to data recorded by the automatic meteo station of Institute of Meteorology and Water Management, located close to experiment. Mean air temperatures were above normal values $(1995-2006)$, from $3.3^{\circ} \mathrm{C}$ (June - December 2006) to $1.4^{\circ} \mathrm{C}$ (2007). Only few months (August 2006, July and September to November of 2007 and September 2008) were slightly colder than normal (ca. $0.1^{\circ}-1^{\circ} \mathrm{C}$ ). Although any water deficits during experiment were reduced with artificial watering, it should be noted that rainfall at 2006 and 2008 were below normal value, $18.6 \mathrm{~mm}$ and $12.9 \mathrm{~mm}$, respectively. During 2007, high rainfall amounts were noted from January to March and from June to July, as contrary to rainfall deficits from April to May and from August to November.

\section{RESULTS}

Climatic conditions as well as genetic properties of tested entries were the major sources of variation for characters measured or calculated (Table 1). Significant effect of entries used in experiment was found for all traits. Shoot dry matter yields were slightly more dependent on identified sources of variation than root dry matter yields. 
Results of three-way ANOVA/MANOVA performed on results obtained in experiment for mature plants (error mean squares)

\begin{tabular}{lccc}
\hline \multirow{2}{*}{ Components of variation } & \multicolumn{2}{c}{ Total biomass of: } & \multirow{2}{*}{ Root / shoot ratio } \\
\cline { 2 - 3 } & Shoots & Roots & 0.003 \\
\hline Years (2007 - 2008) & $1443.90^{* * *}$ & $1467.29^{* * *}$ & $1.432^{* * *}$ \\
Seasons (1-3) & $394.50^{* * *}$ & 144.87 & $1.374^{* * *}$ \\
Entries (15) & $94.32^{* * *}$ & $193.15^{* * *}$ & 0.567 \\
Years $\times$ Seasons & 1.66 & $65.19^{* *}$ & 0.166 \\
Years $\times$ Entries & $31.59^{* *}$ & 46.62 & 0.101 \\
Seasons $\times$ Entries & 11.30 & 11.48 & 0.192 \\
Years $\times$ Seasons $\times$ Entries & 8.92 & 20.70 & \\
\hline
\end{tabular}

$* * *-\mathrm{P}=99 \%, * *-\mathrm{P}=95 \%$

Table 2

Shoots dry mass of tested turf grass varieties and ecotypes (in grams per pot)

\begin{tabular}{lllllllc}
\hline \multirow{2}{*}{$\begin{array}{c}\text { Species., variety name or } \\
\text { ecotype number }\end{array}$} & \multicolumn{1}{c}{ July } & March & July & October & March & July & October \\
\cline { 2 - 8 } DC BROK & $0.35-\mathrm{ABC}$ & $3.11-\mathrm{C}$ & 10.94 & $7.96-\mathrm{B}$ & $14.07-\mathrm{B}$ & $12.92-\mathrm{D}$ & $13.64-\mathrm{C}$ \\
DC POLLBS-236 & $0.40-\mathrm{AB}$ & $5.18-\mathrm{B}$ & 10.47 & $7.69-\mathrm{B}$ & $13.30-\mathrm{B}$ & $12.71-\mathrm{D}$ & $18.26-\mathrm{B}$ \\
FA ASTERIX & $0.36-\mathrm{AB}$ & $4.59-\mathrm{B}$ & 11.71 & $18.52-\mathrm{A}$ & $21.38-\mathrm{A}$ & $24.46-\mathrm{B}$ & $29.03-\mathrm{A}$ \\
FA RAHELA & $0.14-\mathrm{D}$ & $2.89-\mathrm{C}$ & 9.38 & $11.89-\mathrm{AB}$ & $12.27-\mathrm{BC}$ & $23.98-\mathrm{B}$ & $19.35-\mathrm{B}$ \\
FA WOM-100 & $0.37-\mathrm{AB}$ & $5.85-\mathrm{B}$ & 10.47 & $19.66-\mathrm{A}$ & $29.08-\mathrm{A}$ & $30.99-\mathrm{A}$ & $35.04-\mathrm{A}$ \\
FR POLLBS-263 & $0.16-\mathrm{D}$ & $7.89-\mathrm{A}$ & 10.12 & $8.37-\mathrm{B}$ & $12.30-\mathrm{BC}$ & $13.42-\mathrm{D}$ & $16.13-\mathrm{B}$ \\
FR POLLBS-393 & $0.13-\mathrm{D}$ & $5.83-\mathrm{B}$ & 10.52 & $13.11-\mathrm{A}$ & $12.69-\mathrm{BC}$ & $18.72-\mathrm{BC}$ & $26.76-\mathrm{AB}$ \\
FR POLLBS-578 & $0.15-\mathrm{D}$ & $8.93-\mathrm{A}$ & 13.43 & $10.97-\mathrm{B}$ & $13.49-\mathrm{B}$ & $15.89-\mathrm{CD}$ & $22.65-\mathrm{B}$ \\
KM UKR 119 & $0.24-\mathrm{BCD}$ & $3.77-\mathrm{B}$ & 8.51 & $7.84-\mathrm{B}$ & $12.84-\mathrm{BC}$ & $19.36-\mathrm{BC}$ & $16.05-\mathrm{BC}$ \\
LP GATOR & $0.43-\mathrm{AB}$ & $4.16-\mathrm{B}$ & 14.13 & $7.74-\mathrm{B}$ & $8.51-\mathrm{C}$ & $21.43-\mathrm{B}$ & $18.52-\mathrm{B}$ \\
LP LISUNA & $0.42-\mathrm{AB}$ & $3.18-\mathrm{C}$ & 9.45 & $8.91-\mathrm{B}$ & $9.58-\mathrm{C}$ & $21.95-\mathrm{B}$ & $17.74-\mathrm{B}$ \\
LP NUMAN & $0.45-\mathrm{A}$ & $3.94-\mathrm{B}$ & 12.67 & $15.73-\mathrm{A}$ & $10.44-\mathrm{C}$ & $19.39-\mathrm{BC}$ & $21.24-\mathrm{B}$ \\
PP CHALUPY & $0.20-\mathrm{CD}$ & $3.74-\mathrm{BC}$ & 17.45 & $17.73-\mathrm{A}$ & $15.73-\mathrm{B}$ & $17.88-\mathrm{C}$ & $24.74-\mathrm{B}$ \\
PP POLLBS-587 & $0.23-\mathrm{BCD}$ & $2.78-\mathrm{C}$ & 8.18 & $6.02-\mathrm{B}$ & $4.20-\mathrm{C}$ & $11.21-\mathrm{D}$ & $13.82-\mathrm{C}$ \\
PP SKRZESZOWICKA & $0.15-\mathrm{D}$ & $4.36-\mathrm{B}$ & 8.91 & $5.37-\mathrm{B}$ & $5.54-\mathrm{C}$ & $10.72-\mathrm{D}$ & $14.73-\mathrm{C}$ \\
\hline Variation coefficient(CV-\%) & 43 & 38 & 22 & 44 & 46 & 31 & 30 \\
\hline LSD (P=95\%) & 0.18 & 2.11 & n.s. & 7.73 & 8.62 & 6.01 & 9.52 \\
\hline
\end{tabular}

Explanations: DC - Deschampsia caespitosa, FA - Festuca arundinacea, FR - Festuca rubra, KM - Koeleria macrantha, LP - Lolium perenne, KM - Koeleria macrantha, PP- Poa pratensis. A, B, C, ... - means with the same letters are not significantly different $(\mathrm{P}>95 \%)$

\section{Seedling stage}

Growth parameters of tested grass species were already different at the seedling phase (Table2 and 3$)$. The highest seedling shoot dry matter yield (0.35$0.45 \mathrm{~g} / \mathrm{pot}$ ) was noted for Lolium perenne, Festuca arundinacea (excl. Rahela) 
and Deschampsia cespitosa. As contrary to above, only $0.13-0.16 \mathrm{~g} /$ pot was noted for Festuca rubra, F. arundinacea Rahela and Poa pratensis Skrzeszowicka. High variation level was noted also for seedling root system dry weight (Table3). Roots of highest dry matter weight (0.32-0.51 g per pot) were developed by Lolium perenne, Deschampsia caespitosa and Poa pratensis (excl. variety Chałupy). For Koeleria macrantha ecotype, variety Rahela of Festuca arundinacea and two ecotypes of Festuca rubra (POLLBS-578 and POLLBS-393) the lowest root system dry matter weights were noted $(0.2 \mathrm{~g}$ per pot). For other entries values between $0.2-0.3 \mathrm{~g}$ per pot were noted.

Table 3

Root system dry mass of tested turf grass varieties and ecotypes (in grams per pot)

\begin{tabular}{lllllllc}
\hline \multirow{2}{*}{$\begin{array}{c}\text { Species, variety name or } \\
\text { ecotype number }\end{array}$} & \multicolumn{1}{c}{2006} & \multicolumn{2}{c}{2007} & & \multicolumn{2}{c}{2008} \\
\cline { 2 - 8 } DC BROK & $0.33-\mathrm{BC}$ & $3.33-\mathrm{C}$ & $6.12-\mathrm{C}$ & $2.89-\mathrm{D}$ & $4.53-\mathrm{C}$ & $5.62-\mathrm{D}$ & $4.73-\mathrm{F}$ \\
DC POLLBS-236 & $0.32-\mathrm{BC}$ & $4.02-\mathrm{B}$ & $5.29-\mathrm{C}$ & $2.10-\mathrm{D}$ & $5.12-\mathrm{C}$ & $7.21-\mathrm{D}$ & $6.72-\mathrm{E}$ \\
FA ASTERIX & $0.30-\mathrm{BCDE}$ & $4.35-\mathrm{B}$ & $6.81-\mathrm{C}$ & $14.70-\mathrm{B}$ & $22.09-\mathrm{B}$ & $21.15-\mathrm{C}$ & $20.72-\mathrm{D}$ \\
FA RAHELA & $0.19-\mathrm{FG}$ & $5.36-\mathrm{B}$ & $5.27-\mathrm{C}$ & $17.63-\mathrm{B}$ & $23.28-\mathrm{B}$ & $22.75-\mathrm{B}$ & $21.36-\mathrm{D}$ \\
FA WOM-100 & $0.22-\mathrm{DEFG}$ & $3.79-\mathrm{C}$ & $3.97-\mathrm{D}$ & $10.87-\mathrm{C}$ & $20.18-\mathrm{B}$ & $21.27-\mathrm{C}$ & $23.74-\mathrm{D}$ \\
FR POLLBS-263 & $0.37-\mathrm{ABC}$ & $13.45-\mathrm{A}$ & $18.26-\mathrm{A}$ & $19.78-\mathrm{B}$ & $24.56-\mathrm{AB}$ & $25.79-\mathrm{B}$ & $42.20-\mathrm{B}$ \\
FR POLLBS-393 & $0.20-\mathrm{EFG}$ & $12.25-\mathrm{A}$ & $14.61-\mathrm{AB}$ & $30.29-\mathrm{A}$ & $29.29-\mathrm{A}$ & $42.33-\mathrm{A}$ & $52.25-\mathrm{A}$ \\
FR POLLBS-578 & $0.18-\mathrm{FG}$ & $12.17-\mathrm{A}$ & $19.66-\mathrm{A}$ & $27.09-\mathrm{A}$ & $24.90-\mathrm{AB}$ & $23.50-\mathrm{B}$ & $40.19-\mathrm{B}$ \\
KM UKR 119 & $0.13-\mathrm{G}$ & $4.01-\mathrm{BC}$ & $4.72-\mathrm{CD}$ & $7.81-\mathrm{C}$ & $15.46-\mathrm{B}$ & $13.36-\mathrm{C}$ & $13.64-\mathrm{E}$ \\
LP GATOR & $0.46-\mathrm{AB}$ & $5.08-\mathrm{B}$ & $9.24-\mathrm{C}$ & $13.87-\mathrm{B}$ & $13.65-\mathrm{B}$ & $13.82-\mathrm{C}$ & $15.28-\mathrm{D}$ \\
LP LISUNA & $0.37-\mathrm{ABC}$ & $5.07-\mathrm{BC}$ & $9.55-\mathrm{B}$ & $12.77-\mathrm{BC}$ & $12.82-\mathrm{BC}$ & $13.38-\mathrm{C}$ & $10.59-\mathrm{E}$ \\
LP NUMAN & $0.32-\mathrm{BCD}$ & $6.27-\mathrm{B}$ & $6.77-\mathrm{C}$ & $9.19-\mathrm{C}$ & $9.97-\mathrm{C}$ & $15.28-\mathrm{C}$ & $20.83-\mathrm{D}$ \\
PP CHALUPY & $0.26-\mathrm{BCDE}$ & $3.12-\mathrm{C}$ & $18.21-\mathrm{A}$ & $18.22-\mathrm{B}$ & $38.13-\mathrm{A}$ & $23.54-\mathrm{B}$ & $29.20-\mathrm{C}$ \\
PP POLLBS-587 & $0.51-\mathrm{A}$ & $4.52-\mathrm{B}$ & $7.38-\mathrm{C}$ & $9.07-\mathrm{C}$ & $16.61-\mathrm{B}$ & $14.83-\mathrm{C}$ & $22.22-\mathrm{D}$ \\
PP SKRZESZOWICKA & $0.39-\mathrm{AB}$ & $3.21-\mathrm{C}$ & $11.58-\mathrm{B}$ & $16.30-\mathrm{B}$ & $12.66-\mathrm{BC}$ & $13.34-\mathrm{C}$ & $17.82-\mathrm{D}$ \\
\hline Variation coefficient(CV-\%) & 35 & 59 & 54 & 55 & 50 & 48 & 59 \\
\hline LSD (P=95\%) & 0.12 & 1.26 & 5.24 & 7.25 & 12.57 & 10.11 & 9.11 \\
\hline
\end{tabular}

Explanations: DC - Deschampsia caespitosa, FA - Festuca arundinacea, FR - Festuca rubra, KM - Koeleria macrantha, LP - Lolium perenne, KM - Koeleria macrantha, PP- Poa pratensis. A, B, C, ... - means with the same letters are not significantly different $(\mathrm{P}>95 \%)$

Root systems of seedlings of Poa pratensis variety Skrzeszowicka, ecotype POLLBS-587 and Festuca rubra ecotype POLLBS-263 were found to be heavier than shoots more than twice (Table 4). For the rest of tested entries root:shoot ratios were close to or lower than 1.0. The lowest values of seedlings root:shoot ratio $(0.5-0.7)$ were calculated for Koleria macrantha, Festuca arundinacea variety WOM 100 and Lolium perenne variety Numan. 
Root to shoot ratio of tested turf grass varieties and ecotypes

\begin{tabular}{|c|c|c|c|c|c|c|c|}
\hline \multirow{2}{*}{$\begin{array}{l}\text { Species., variety name or } \\
\text { ecotype number }\end{array}$} & \multicolumn{2}{|l|}{2006} & \multicolumn{2}{|l|}{2007} & \multicolumn{3}{|c|}{2008} \\
\hline & July & March & July & October & March & July & October \\
\hline DC BROK & $0.9-\mathrm{D}$ & $1.1-\mathrm{B}$ & $0.6-\mathrm{C}$ & $0.4-\mathrm{C}$ & $0.3-\mathrm{C}$ & $0.4-\mathrm{D}$ & $0.3-\mathrm{E}$ \\
\hline DC POLLBS-236 & $0.8-\mathrm{D}$ & $0.8-\mathrm{B}$ & $0.5-\mathrm{C}$ & $0.3-\mathrm{C}$ & $0.4-\mathrm{C}$ & $0.6-\mathrm{C}$ & $0.4-\mathrm{E}$ \\
\hline FA ASTERIX & $0.8-\mathrm{D}$ & $0.9-\mathrm{B}$ & $0.6-\mathrm{C}$ & $0.8-\mathrm{BC}$ & $1.0-\mathrm{C}$ & $0.9-\mathrm{C}$ & $0.7-\mathrm{D}$ \\
\hline FA RAHELA & 1.4-BCD & 1.9-A & $0.6-\mathrm{C}$ & $1.5-\mathrm{B}$ & 1.9-BC & $0.9-\mathrm{C}$ & $1.1-\mathrm{CD}$ \\
\hline FA WOM-100 & $0.6-\mathrm{D}$ & $0.6-\mathrm{B}$ & $0.4-\mathrm{C}$ & $0.6-\mathrm{C}$ & $0.7-\mathrm{C}$ & $0.7-\mathrm{C}$ & $0.7-\mathrm{D}$ \\
\hline FR POLLBS-263 & $2.3-\mathrm{BC}$ & $1.7-\mathrm{A}$ & $1.8-\mathrm{A}$ & 2.4-A & $2.0-\mathrm{B}$ & $1.9-\mathrm{AB}$ & $2.6-\mathrm{A}$ \\
\hline FR POLLBS-393 & $1.5-\mathrm{BC}$ & 2.1-A & 1.4-B & 2.3-A & 2.3-B & $2.3-\mathrm{A}$ & $1.9-\mathrm{B}$ \\
\hline FR POLLBS-578 & $1.2-\mathrm{CD}$ & $1.4-\mathrm{AB}$ & $1.5-\mathrm{AB}$ & $2.5-\mathrm{A}$ & $1.8 \mathrm{C}$ & $1.5-\mathrm{B}$ & $1.8-\mathrm{B}$ \\
\hline KM UKR 119 & $0.5-\mathrm{D}$ & $1.1-\mathrm{B}$ & $0.6-\mathrm{C}$ & $1.0-\mathrm{BC}$ & $1.2-\mathrm{C}$ & $0.7-\mathrm{C}$ & $0.8-\mathrm{D}$ \\
\hline LPGATOR & $1.1-\mathrm{CD}$ & $1.2-\mathrm{AB}$ & $0.7-\mathrm{C}$ & $1.8-\mathrm{B}$ & $1.6-\mathrm{C}$ & $0.6-\mathrm{C}$ & $0.8-\mathrm{D}$ \\
\hline LP LISUNA & $0.9-\mathrm{D}$ & $1.6-\mathrm{A}$ & $1.0-\mathrm{C}$ & 1.4-B & $1.3-\mathrm{C}$ & $0.6-\mathrm{C}$ & $0.6-\mathrm{D}$ \\
\hline LP NUMAN & $0.7-\mathrm{D}$ & $1.6-\mathrm{A}$ & $0.5-\mathrm{C}$ & $0.6-\mathrm{C}$ & $0.9-\mathrm{C}$ & $0.8-\mathrm{C}$ & $1.0-\mathrm{D}$ \\
\hline PPCHALUPY & 1.3-BCD & $0.8-\mathrm{B}$ & $1.0-\mathrm{C}$ & $1.0-\mathrm{BC}$ & 2.4-B & $1.3-\mathrm{B}$ & $1.2-\mathrm{C}$ \\
\hline PP POLLBS-587 & 2.2-B & $1.6-\mathrm{A}$ & $0.9-\mathrm{C}$ & $1.5-\mathrm{B}$ & 3.9-A & $1.3-\mathrm{B}$ & $1.6-\mathrm{B}$ \\
\hline PP SKRZESZOWICKA & 2.6-A & $0.7-\mathrm{B}$ & $1.3-\mathrm{B}$ & $3.0-\mathrm{A}$ & 2.3-B & $1.2-\mathrm{BC}$ & $1.2-\mathrm{C}$ \\
\hline Variation coeff. (CV - \%) & 51 & 36 & 48 & 63 & 59 & 51 & 56 \\
\hline $\operatorname{LSD}(\mathrm{P}=95 \%)$ & 0.80 & 0.75 & 0.33 & 1.11 & 1.05 & 0.36 & 0.53 \\
\hline
\end{tabular}

Explanations: DC - Deschampsia caespitosa, FA - Festuca arundinacea, FR - Festuca rubra, KM - Koeleria macrantha, LP - Lolium perenne, KM - Koeleria macrantha, PP- Poa pratensis. A, B, C, ... - means with the same letters are not significantly different $(\mathrm{P}>95 \%)$

\section{Mature plants - first year}

Differences between entries were also found during further growth and development. The highest shoot biomass after first year of observations was developed by Festuca arundinacea (average $10.6 \mathrm{~g}$ per pot, total from three seasons - $31.7 \mathrm{~g}$ ). However, high shoot biomass was not followed by high root biomass. For Festuca arundinacea average root biomass was only $8.1 \mathrm{~g}$ per pot (total from three seasons $24.3 \mathrm{~g}$ ) and it was lower than for Lolium perenne (8.6 g and $25.9 \mathrm{~g}$, respectively), Poa pratensis (10.2 g and $30.5 \mathrm{~g}$, respectively), and Festuca rubra (18.6 g and $55.9 \mathrm{~g}$, respectively). The lowest values of both shoot and root biomass were noted for Deschampsia cespitosa and Koeleria macrantha. Root:shoot ratios were also different for tested entries since the first year of observations. It was the highest during all seasons for only one entry - ecotype POLLBS-263 of Festuca rubra but also high during spring and autumn for next ecotype from mentioned species - POLLBS-393. Average root:shoot ratio was the highest for Festuca rubra (1.9) and also high for Poa pratensis and Lolium perenne (1.3 for both). The lowest average value of root:shoot ratio (0.5) were found for ecotype POLLBS-36 of Deschampsia cespitosa and variety WOM100 of Festuca arundinacea, which produced very high amount of shoot biomass ( $36.0 \mathrm{~g}$ per year). 


\section{Mature plants - second year}

In the second year of experiment, higher differences between tested entries and species were observed. Like in the 2007, the highest shoot dry mass was developed by Festuca arundinacea (average - 25.2 g per pot, total from three seasons - 225.6 g). Variety WOM100 from mentioned species produced significantly the highest shoot biomass during all seasons, and variety Asterix at spring and autumn. Contrary to above, Poa pratensis varieties and ecotype produced the average lowest shoot yields (average $13.2 \mathrm{~g}$, yearly total $118.6 \mathrm{~g}$ ). Similar relations as year before were noted for root biomass. It was the highest for Festuca rubra, with ecotype POLLBS05-393 ranked the highest among all entries during all seasons. Total yearly produced root biomass by mentioned ecotype $(123.9 \mathrm{~g})$ was 8.3 times greater then the lowest root biomass, produced by Deschampsia cespitosa variety Brok (14.9 g). The same relation calculated for yearly shoot biomass was only 1.4. Considering root:shoot ratio, the highest values were noted also for Festuca rubra (species yearly average - 2.0)

Table 5.

Correlation coefficients between seedling traits and mature plants characteristics

\begin{tabular}{|c|c|c|c|c|c|c|c|}
\hline \multirow{2}{*}{ Year } & \multirow{2}{*}{ Season } & \multirow{2}{*}{ Mature plants traits } & \multicolumn{5}{|c|}{ Correlation coefficients for seedling trais: } \\
\hline & & & \multicolumn{2}{|c|}{ Shoot, dry mass } & \multirow{2}{*}{$\begin{array}{c}\text { Root, dry mass } \\
-0.19\end{array}$} & \multicolumn{2}{|c|}{ Root:/shoot ratio } \\
\hline & & Root system, dry mass & -0.46 & & & 0.30 & \\
\hline & Spring & Shoots, dry mass & -0.32 & & -0.28 & 0.14 & \\
\hline & & Root:shoot ratio & -0.31 & & 0.01 & 0.26 & \\
\hline & & Root system, dry mass & -0.57 & & -0.04 & 0.48 & \\
\hline \multirow[t]{9}{*}{2007} & Summer & Shoots, dry mass & 0.13 & & -0.08 & -0.24 & \\
\hline & & Root:shoot ratio & -0.66 & $* *$ & 0.09 & 0.71 & $* * *$ \\
\hline & & Root system, dry mass & -0.67 & $* *$ & -0.31 & 0.38 & \\
\hline & Autumn & Shoots, dry mass & 0.16 & & -0.45 & -0.46 & \\
\hline & & Root:shoot ratio & -0.68 & $* *$ & 0.12 & 0.75 & $* * *$ \\
\hline & & Root system, dry mass & -0.62 & $* *$ & -0.38 & 0.22 & \\
\hline & Spring & Shoots, dry mass & 0.20 & & -0.53 & -0.56 & \\
\hline & & Root:shoot ratio & -0.62 & $* *$ & 0.33 & 0.75 & $* * *$ \\
\hline & & Root system, dry mass & -0.58 & $* *$ & -0.41 & 0.23 & \\
\hline \multirow[t]{8}{*}{2008} & Summer & Shoots, dry mass & 0.32 & & -0.41 & -0.61 & $* *$ \\
\hline & & Root:shoot ratio & -0.77 & $* * *$ & -0.09 & 0.65 & $* *$ \\
\hline & & Root system, dry mass & -0.65 & $* *$ & -0.29 & 0.39 & \\
\hline & Autumn & Shoots, dry mass & 0.1 & & -0.47 & -0.41 & \\
\hline & & Root:shoot ratio & -0.72 & $* * *$ & 0.01 & 0.69 & $* * *$ \\
\hline & \multirow{3}{*}{ Average } & Root system, dry mass & -0.68 & & -0.33 & 0.37 & \\
\hline & & Shoots, dry mass & 0,19 & $* *$ & -0.51 & -0.54 & $* *$ \\
\hline & & Root:shoot ratio & -0.76 & $* * *$ & 0.13 & 0.79 & $* * *$ \\
\hline
\end{tabular}

$* * *-\mathrm{P}=99 \%, * *-\mathrm{P}=95 \%$ 
In case of Festuca arundinacea, Poa pratensis, Deschampsia cespitosa and Koeleria macrantha increase (ca. 50 - 97\%) of root dry matter measured in spring of 2008 as compared to autumn of 2007 was found. Increase of root dry matter during autumn and winter was only minor for Festuca rubra and Lolium perenne (ca. 2\%).

Shoot dry matter increases between October 2007 and March 2008 were from 63.7 - 74.7\% for Deschampsia caespitosa and Koeleria macrantha but from 25.0 - 26.0\% for both Festuca species. Reverse relation was found for Poa pratensis and Lolium perenne, where decrease of ca. $12 \%$ was noted in mentioned period.

\section{Relations between seedlings and mature plants}

For seedling shoot dry mass and seedling root:shoot ratio significant correlation coefficients with mature plants traits were calculated for all seasons (excl. March 2007) (Table 5). The highest correlation coefficient was calculated for seedling root:shoot ratio and mature plants root:shoot ratio averaged across all seasons $(\mathrm{r}=0.79, \alpha>0.001)$. Above relation is true only for all tested entries, however similar trends were found also for entries in particular species: Festuca arundinacea, Lolium perenne, Poa pratensis and Festuca rubra ( Fig. 1).

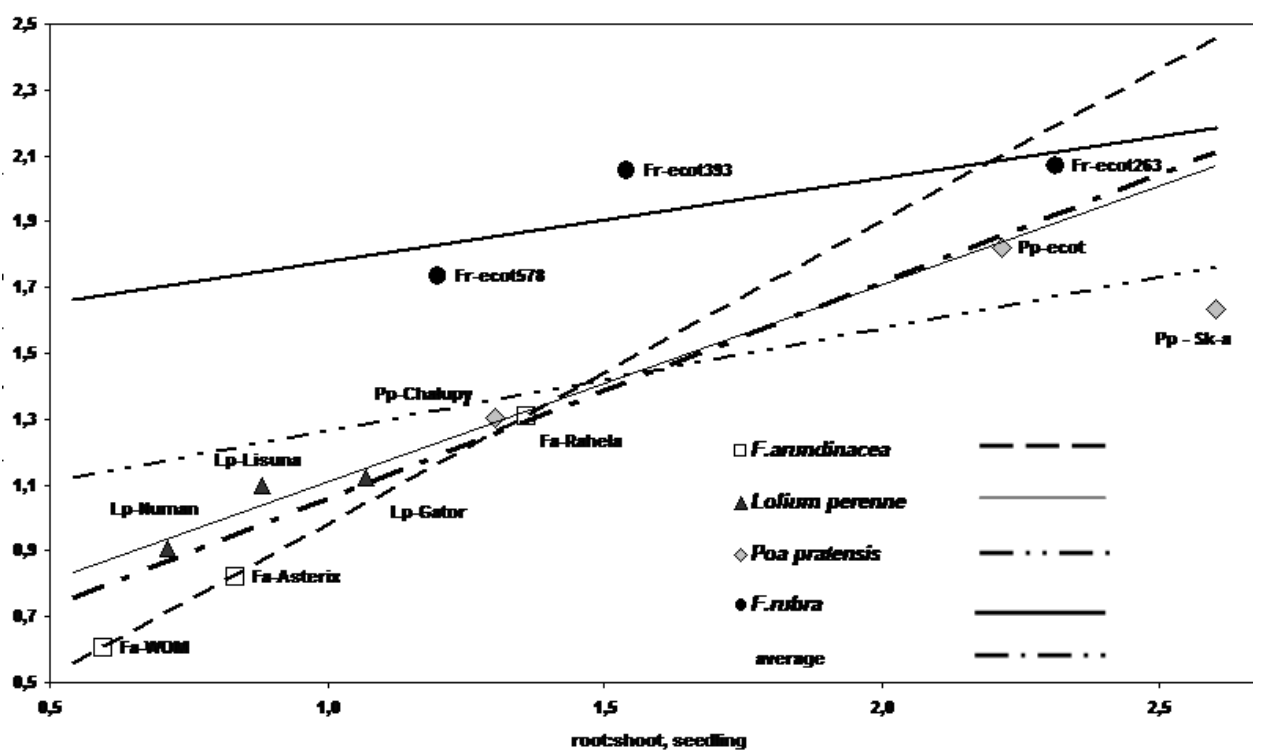

Fig. 1. Relation between root:shoot ratio at seedling stage and at mature plants (average from two years and 3 seasons per year)

Values of root:shoot rations observed at seedling phase are almost the same as for mature plants (averaged value) for Festuca arundinacea varieties. In case of two tested entries of Poa pratensis and all of Deschampsia cespitosa, seedling root:shoot values were higher as compared to mature plants values. For 
Festuca rubra aforementioned relation was found for only one entry. For Lolium perenne varieties and Koeleria macrantha ecotype root:shoot ratios at seedling phase were lower than averaged values for mature plants.

\section{DISCUSSION}

Initial seedling growth rate and dimension of above- and belowground biomass were specific for species as well as varieties and ecotypes used. Similar high differentiation at initial stage of growth of turf grasses was described by others (Harkot 1998, Harkot 2006, Żurek 2004, Harkot and Czarnecki 1998). However, mentioned relations were the effect of different factors as time of sowing, frequency of mowing, natural and simulated drought, soil fertility or turf dry-out. We have made our conclusions on the basis of uniform soil, water and fertilization conditions. Generally, the extent of root system is correlated with above-ground growth (Gibson 2009). It is however, not quite sure at the seedling phase. On the basis of our results Lolium perenne, Deschampsia cespitosa and Festuca arundinacea shoots yielded much better then roots. Similar conclusions concerning seedling dimensions were given by Harkot and Czarnecki (1998) and Czarnecki and Harkot (2003). It has been concluded by Kittock and Pattersona (1962) that seedling size is the effect of seed size. Due to TSW data given for example by Falkowski (1982) it is questionable in case of Deschampsia cespitosa, species of very small seed size (TSW $=0.2-0.3 \mathrm{~g}$ ), yielding seedlings of size comparable to Lolium perenne (TSW=1.3 - $2.5 \mathrm{~g}$ ). Tufted hairgrass (Deschampsia caespitosa) is quite interesting species with large seedling and lowest root system with coincident high shoot growth. According to Żurek (1999) and Prończuk et. al. (2001) mentioned species is of great potential for extensive, shaded and sunny grass areas. Our results suggest that relatively small root system is able to regenerate during autumn and winter. Relatively good ability to regenerate could be the effect of natural, high chlorophyll contents in Deschampsia leaves (Falkowski 1982).

Root:shoot ratio is very important in defense against stress conditions, for example drought. Festuca rubra, due to it's ability to increase root:shoot ratio with decreasing water availability (reduction of above ground biomass with extensive root growth) is one from drought resistant species (Żurek, 2006, Dziamski et al. 2007).

\section{CONCLUSIONS}

1. Proportion of biomass allocated below and above ground varies among species, varieties or ecotypes at the seedling phase as well as 2.5 years old plants. 
2. Specific genetic properties affect different biomass allocation. Festuca rubra and Poa pratensis produced relatively heavier root system as compared to shoots but for other species (Festuca arundinacea, Lolium perenne, Deschampsia cespitosa, Koeleria macrantha) mentioned relation was quite reverse.

3. Root : shoot ratio at seedling stage seems to be a good indicator of root:shoot values for mature plants.

4. Selection for high root:shoot values are mostly promising in Poa pratensis and Festuca rubra, where relatively high differences of root:shoot values at seedling stage among entries were noted.

\section{REFERENCES}

Czarnecki Z., Harkot W. 2003. Ocena tempa odrastania gazonowych odmian Lolium perenne L. Biul. IHAR. 225. 289-294.

Dziamski A., Stypczyńska Z., Żurek G., Łabędzki L., Długosz J. 2007. Observations of root system development and dynamics of root:shoot ratio of selected turf grass varieties and breeding lines grown in different soil conditions. Plant Breeding and Seed Science, 55: 76-88.

Falkowski M. 1982. Trawy polskie. PWRiL, Warszawa. pp. 566.

Gibson D.J. 2009. Grasses and Grassland Ecology. Oxford University Press. pp. 305.

Grabowski K., Grzegorczyk S., Bendycki S., Kwietniewski H. 1999 a. Ocena wartości użytkowej wybranych gatunków i odmian traw gazonowych do obsiewu nawierzchni trawiastych. Fol. Univ. Agric. Stetin. 197 Agricultura (75). 81-88.

Grabowski K., Grzegorczyk S., Bendycki S., Kwietniewski H. 1999 b. Przydatność gatunków i odmian traw gazonowych do obsiewu trawników rekreacyjnych. Fol. Univ. Agric. Stetin.. 197 Agricultura (75). 8952.

Gross K.L., Maruca D., Pregitzer K.S. 1992. Seedling growth and root morphology of plants with different life-histories. New Phytol. 120. 535 - 542.

Harkot W. 1998. Dynamika początkowego wzrostu i rozwoju traw jako kryterium ich przydatności do podsiewu. Łąkarstwo w Polsce. 1. 139-146.

Harkot W. 2006. Wschody i instalacja wybranych odmian traw gazonowych w różnych terminach siewu nasion. Zesz. Nauk. Uniw. Przyr. we Wrocławiu. Roln. LXXXVIII. 545. 111-120.

Harkot W., Czarnecki Z. 1998. Dynamika wydłużania systemu korzeniowego siewek polskich odmian traw gazonowych. Ann. Univ. MCS. Sectio E. LIII. 20. 177-185.

Jankowski K., Jodełka J., Ciepiela G., Kolczarek R. 2003. Ocena traw gazonowych w ekstensywnym użytkowaniu trawnika. Biul. IHAR. 225. 259-264.

Kittock D.L., Patterson J.K. 1962. Seed size effect on the performance of dryland grasses. Agron. J. 54: $277-$ 278.

Prończuk S. 1993. System oceny traw gazonowych. Biul. IHAR. 186. 127-131.

Prończuk S. 1994. Stan hodowli i nasiennictwa traw gazonowych w Polsce. Genetica Polonica. 35A. 329 339.

Prończuk M., Prończuk S., Żebrowski J. 2003. Response of Lolium perenne cultivars and ecotypes to artificial and natural shade conditions. Czech J. Genet. Plant Breed., 39: 363 - 366.

Prończuk S., Prończuk M., Żyłka D. 1997. Metody syntetycznej oceny wartości użytkowej traw gazonowych. Zesz. Problem. Post. Nauk Roln. 451. 125-133.

Prończuk S., Żurek G., Żyłka D., Prończuk M. 2001. Ocena śmiałka darniowego (Deschampsia caespitosa L.) w różnym użytkowaniu trawnikowym. Zesz. Problem. Post. Nauk Roln. 474. 113-121.

Rutkowska B., Hempel A. 1986. Trawniki. PWRiL Warszawa. 256.

Rutkowska B., Pawluśkiewicz M. 1996. Trawniki. Poradnik zakładania i pielęgnowania. PWRiL. Warszawa. pp. 101.

Sherman R.C. 2006. Fifty years of splendor in the grass. Crop Sci. 46. $2218-2229$.

Stuczyńska J., Jakubowski S. 1980. Wpływ częstotliwości koszenia na ilość i jakość masy korzeni niektórych traw po dwuletnim użytkowaniu. Biul. IHAR. 140. 34-46. Tołkwińska M. 1975. Dynamika przyrostu masy podziemnej roślinności łąkowej w zależności od nawożenia. Roczn. Glebozn. t. XXVI. z. 3. 245 258. 
Waddington D.V., Carrow R.N., Sherman R.C. 1992. Turfgrass. Agronomy Series, no. 32. American Soc. of Agronomy, Crop Science Soc. of America, Soil Science Soc.of America, Madison, Wisconsin, USA. pp. 805.

Żurek G. 1999.Evaluation and characterization of wild ecotypes of tufted hairgrass (Deschampsia caespitosa L.) collected from East Poland. ECP/GR Forages working group: Seventh Meeting. Portugal: 208-212.

Żurek G. 2004. Reakcja wybranych odmian traw gazonowych na naturalną i symulowaną suszę. Biul. IHAR, 233: 195-209.

Żurek G. 2006. Reakcja traw na niedobory wody - metody oceny i ich zastosowanie dla gatunków trawnikowych. Monogr. i Rozpr. Nauk IHAR, 25: pp 106.

Żurek G. 2008.The relationship between sod strength and turf quality of common grass cultivars. Plant Breeding and Seed Science, 57: $25-34$

Żurek G., Prończuk S., Żyłka D. 2001. Ocena przydatności ekotypów wiechliny łąkowej (Poa pratensis L.) do warunków intensywnego użytkowania trawnikowego Zesz. Problem. Post. Nauk Roln.. 474. 139-143. 